\title{
Représentations de la violence envers les femmes dans le couple: mesures du phénomène - Le cas français
}

1

Dominique Fougeyrollas-Schwebel - FRANCE

Chercheuse, Institut de recherche interdisciplinaire en sociologie, économie et science politique,

Centre national de la recherche scientifique, Université Paris-Dauphine

Maryse Jaspard - FRANCE

Chercheuse, Institut national d'études démographiques, Laboratoire Démographie, Genre et Sociétés

\section{RÉSUMÉ}

En dépit des progrès dans l'égalité des droits entre les deux sexes, la domination masculine perdure dans notre société. Au sein des couples, cette domination se manifeste par des actes de violence émanant principalement des hommes. L'Enquête nationale sur les violences envers les femmes en France (ENVEFF - 2000) a été menée dans l'objectif de cerner, par une analyse quantitative, les violences envers les femmes. Cette communication retrace en premier lieu l'évolution du contexte institutionnel et social, notamment des mouvements féministes, dans la prise en compte de la question des violences envers les femmes. Elle décrit le paysage dans lequel l'enquête ENVEFF a dû se construire. Est ensuite abordé l'impact de cette enquête, en particulier les évolutions les plus récentes pour chiffrer le phénomène des violences.

En 2007, l'Institut national de la statistique et des études économiques (INSEE) a pour la première fois élargi l'enquête Cadre de vie et sécurité sur les faits de violences (vols, coups) aux violences conjugales et aux violences sexuelles. En près de dix ans, la reconnaissance des violences comme fait de société ainsi que la médiatisation accrue du phénomène semblent avoir permis à davantage de femmes de dénoncer les violences subies. Mais la mise en place de dispositifs statistiques institutionnels n'est pas sans conséquences sur la perception du phénomène.
ABSTRACT Despite the progress achieved in the area of equal rights between men and women, male domination still endures in our society. Within couples, this domination is manifested through acts of violence perpetrated mainly by men. The French national survey on violence against women (ENVEFF - 2000) was conducted to determine, through a quantitative analysis, the violent acts committed against women. This article first relates the evolving institutional and social context, in particular the feminist movements, in taking account of the issue of violence against women. It describes the landscape in which the ENVEFF survey was developed. The impact of this survey is then examined, in particular the most recent developments in terms of assessing the phenomenon of violence.

In 2007, the French National Institute for Statistics and Economic Studies (INSEE) for the first time broadened the Living Environment and Safety Survey to include not only violent acts (thefts, blows), but also spousal violence and sexual violence. Over this last decade, the recognition of violent acts as a societal fact and the increased mediatization of this issue seem to have led more women to denounce the violence they have experienced. However, the establishment of institutional statistical mechanisms has also had an effect on the perception of this phenomenon. 
L a reconnaissance des violences contre les femmes et leur dénonciation au plan social et judiciaire, la possibilité pour les femmes de porter plainte contre les violences sexuelles, y compris conjugales, sont un des faits marquants de notre modernité, d'une modernité portée en l'occurrence par la «deuxième vague» des mouvements féministes.

$\mathrm{Au}$ cours des années 70 émerge une approche nouvelle des violences contre les femmes. Les analyses insistent sur les dimensions institutionnelles de ces violences et non pas simplement sur le caractère interindividuel d'une gestion défaillante des conflits entre personnes; les facteurs liés aux caractères psychopathologiques de non-maîtrise de soi sont minimisés au profit de l'analyse des dispositifs de pouvoir, montrant ainsi que les femmes sont très majoritairement victimes des violences conjugales et des violences sexuelles. En dépit des progrès dans l'égalité des droits entre les deux sexes, la domination masculine perdure dans notre société. Au sein des couples, cette domination se manifeste par des actes de violence émanant principalement des hommes.

Cependant, considérer que les violences conjugales sont un des aspects, sans doute le plus caché, du continuum des violences exercées à l'encontre des femmes est loin de faire consensus. Aujourd'hui, la conceptualisation de ces violences évolue entre la tentation de réduire les violences envers les femmes aux seules violences conjugales et une réticence à associer violences et vie conjugale, particulièrement à notre époque où la relation de couple est réputée librement choisie et considérée comme espace de liberté et d'épanouissement individuel.

\section{Le mouvement féministe, le droit et la prise en compte des violences contre les femmes}

En 1970, les dernières modifications du Code civil proclament l'égalité juridique des époux, mais les droits spécifiques des femmes avancent lentement comparativement à d'autres pays, comme la Grande-Bretagne notamment. En 1967, la loi Neuwirth autorise la contraception, mais les décrets d'application s'échelonnent jusqu'en 1974 et il faudra attendre 1979 pour que la loi autorisant l'interruption volontaire de grossesse (IVG), sous conditions, soit votée. Cependant les évolutions démographiques se font jour dès les années 60 : baisse de la fécondité, déclin du mariage, développement de la cohabitation, accroissement des naissances hors mariage, montée du divorce. Les femmes ont désormais acquis une relative autonomie par rapport à la procréation, ainsi qu'au plan économique grâce à leur présence sur le marché du travail et à l'élévation de leur niveau d'études. Néanmoins, au sein de l'univers familial, le partage du travail domestique reste bien inégal.

La mobilisation féministe sur les questions des violences sexuelles, qui avait émergé dès 1976 afin de faire reconnaître le viol comme crime, aboutit en 1980 à de nouvelles formulations du Code pénal. Les violences conjugales ont été prises en compte en tant qu'infractions spécifiques dans le nouveau Code pénal de 1992, en application depuis 1994 sous la qualification de circonstances aggravantes. Au demeurant, si des législations égalitaires ont été promues, il faudra encore attendre pour constater un mouvement équivalent dans les pratiques judiciaires (Fougeyrollas-Schwebel, Jaspard, 2003).

À partir de 1992, la mobilisation pour la parité en politique contribue à une plus forte reconnaissance des discriminations à l'encontre des femmes et les campagnes contre les violences faites aux femmes s'inscrivent dans un contexte où les revendications des mouvements féministes jouissent d'un préjugé beaucoup plus favorable. La quatrième Conférence mondiale de l'ONU sur les femmes (Pékin, septembre 1995) marque un tournant dans la mise en œuvre des politiques de lutte contre les violences faites aux femmes. Désormais, les pouvoirs publics renforcent les programmes de lutte contre ces violences en raison de l'obligation faite à la France de tenir son rang au sein des organisations internationales. Ainsi, en 1997, le Service des droits des femmes commanditait une Enquête nationale sur les violences envers les femmes en France (ENVEFF) (Jaspard et al., 2003) afin de cerner l'ampleur et la nature du phénomène pour mieux adapter les politiques de prévention et les actions d'aide aux victimes.

À l'aube du troisième millénaire, et avec la publication des résultats de l'enquête 
ENVEFF, la question des violences contre les femmes entre dans le débat public et politique, y compris dans les programmes des candidats à la présidence de la République; les réformes législatives suivront. Après les modifications de la loi sur le divorce en 2004, le retard français est finalement rattrapé par la loi du 4 avril 2006 qui renforce la prévention et la répression des violences au sein du couple. Elle élargit le champ d'application des circonstances aggravantes aux pacsés ${ }^{1}$ et aux «ex», de même qu’à de nouvelles infractions (meurtres, viols, agressions sexuelles); elle facilite l'éloignement de l'auteur de l'infraction du domicile de la victime; elle reconnait le viol entre époux dans le but d'assujettissement de la victime. Cette loi qualifie explicitement le viol conjugal, jusqu'ici soumis à la jurisprudence. Désormais, la présomption de consentement des époux aux actes sexuels ne vaut que jusqu’à preuve du contraire.

\section{Définition et mesure des violences conjugales: enjeu scientifique et politique}

\section{La première enquête: \\ I'enquête ENVEFF}

L'enquête ENVEFF (voir encadré p. 114), première enquête nationale sur ce thème réalisée en France, fait partie du programme d'enquêtes statistiques des services publics établi par le Conseil national de l'information statistique pour l'année 2000 (Journal officiel du 23 janvier 2000). Cette enquête dresse un panorama des divers types d'atteintes et de violences interpersonnelles (verbales, psychologiques, physiques et sexuelles) que l'ensemble des femmes ont pu subir dans leurs cadres de vie (espaces publics, travail, couple et famille), quels qu'en soient les auteurs. En l'absence d'antériorité méthodologique et conceptuelle adaptée au contexte français, les chercheurs de l'enquête ENVEFF ont dû innover sur ces deux plans. Cette recherche pionnière demeure une référence dans le domaine des violences contre les femmes - aussi bien pour la recherche que les institutions et les associations.

Les premiers résultats de l'enquête ont été largement diffusés par les médias. Une forme de consensus s'est opérée pour faire de la violence envers les femmes un problème de société, mais la réflexion scientifique sur le thème s'est peu développée. Un chiffre a plus particulièrement retenu l'attention: une femme sur dix est victime de violences conjugales. L'ampleur de ce chiffre a surpris et sa divulgation a accéléré la prise de conscience de l'ensemble du corps social (Chetcuti, Jaspard, 2007). La campagne de lutte contre les violences envers les femmes, mise en œuvre par le Secrétariat aux droits des femmes en 2001, s'est inspirée des conclusions de l'enquête ENVEFF et a intégré le changement d'image de femmes victimes de violences: de «la femme battue» (l'autre, stigmatisée), on est passé à une «femme victime de violences» (soi-même ou une proche), qui se tait, mais qui est incitée à parler.

\section{Distinguer la violence du conflit}

Une des difficultés de l'approche des violences conjugales est de distinguer les actes de violence des actes de conflit. Le terme «violences conjugales » évoque la plupart du temps une relation de couple violente qui se manifeste par des scènes de ménage. Il peut arriver que des interactions entre les partenaires puissent prendre des formes agressives. Si le conflit est, en quelque sorte, inhérent à la vie sociale, la violence s'en distingue par la volonté d'emprise sur l'autre et le désir de destruction. Violence et agressivité ne peuvent être assimilées; et maintenir la confusion entre les deux fait partie des tentatives de «naturaliser» la violence des hommes pour la rendre socialement légitime.

L'enquête ENVEFF a écarté l'analyse en termes d'échelle de conflit au profit d'une étude distinguant les situations de violence des situations de conflit ${ }^{2}$.

1. Personnes ayant conclu un pacte civil de solidarité (PACS). Instauré par la loi n 99-944 du 15 novembre 1999 sous le gouvernement Jospin, le PACS est un contrat passé entre deux personnes majeures, de sexe différent ou de même sexe, pour organiser leur vie commune. Cette loi est née d'une volonté de comble le vide juridique entourant les couples non mariés. Le PACS est destiné aux personnes qui désirent vivre ensemble mais qui ne veulent ou ne peuvent se marier, aux couples hétérosexuels ou homosexuels. Dispositif universel, il apporte une sécurité juridique minimale, se situant entre le concubinage (statut flou mais garant d'une certaine liberté) et le mariage.

2. Le mode d'approche et le cheminement du questionnaire ont été conçus pour éviter la confusion. La première partie de l'entretien comporte des questions sur les disputes répétées au sein du couple et sur le mode de partage du travail domestique. Les questions de violence ne sont abordées qu'ensuite. 


\section{Repérer les situations de violence conjugale}

Dans la recherche de l'enquête ENVEFF, la notion de conjugalité est prise dans un sens large, elle s'étend à toute relation de couple, avec ou sans lien légal, avec ou sans cohabitation. L'analyse des violences dans la sphère privée s'est ainsi adaptée aux évolutions de la conjugalité: des violences domestiques ou intra-familiales aux violences perpétrées par un partenaire intime.

Pour mesurer la violence conjugale, distinguer séparément des types de violences verbales, psychologiques, physiques ou sexuelles s'avérait peu significatif; c'est moins la nature des agressions que leur répétition, voire leur cumul, qui détermine la gravité des situations de violence. L'enquête ENVEFF a proposé une mesure des violences au sein du couple en calculant un taux global de femmes «en situation de violence conjugale» qui combinait l'ensemble des types de violence en tenant compte du nombre des faits cités et de leur fréquence. Ce taux est subdivisé en deux niveaux afin de montrer la progression de la gravité des situations. Le niveau «grave» correspondant aux insultes répétées et au harcèlement psychologique et, dans des cas plus rares, à des agressions physiques ou sexuelles uniques $(7 \%)$. Le niveau «très grave» regroupe les situations de cumul de violences; ici se produisent souvent des agressions physiques ou sexuelles, répétées ou associées aux violences verbales et au harcèlement psychologique (2,5\%). Lusage d'un indicateur de ce type a permis des analyses fines des contextes sociologiques et des processus de violences conjugales. Il ressort que l'appartenance sociale est un facteur peu discriminant alors que les situations d'exclusion comme le chômage sont des facteurs aggravants (Jaspard et al. 2003).

\section{Évolutions de la prise en compte des violences conjugales}

\section{Les violences psychologiques: composante principale \\ des violences conjugales}

L’enquête ENVEFF a révélé l'importance des violences psychologiques chez les couples en situation de violence conjugale. Outre les insultes et les agressions verbales, un ensemble de questions permettaient de cerner les atteintes psychologiques et les situations de harcèlement (menaces, contrôle, jalousie, dénigrement, dévalorisation, chantage affectif).

En 2003, dans un contexte de dénonciation du harcèlement moral dans les sphères privée et publique et d'évolutions législatives sur le harcèlement moral au travail, la virulence de la polémique lancée par Élisabeth Badinter contre la prise en compte des violences psychologiques comme composante des violences conjugales cherchait à discréditer les associations d'aide aux victimes ${ }^{3}$. En 2008, elles doivent toujours plaider pour la reconnaissance du harcèlement psychologique comme constitutif de la violence conjugale et mode de domination d'un sexe sur l'autre. Si les violences physiques sont réprouvées, les violences psychologiques sont plus rarement prises en compte; il est plus difficile d'en faire la preuve et les victimes ne les perçoivent pas toujours.

Bien qu'une certaine évolution s'amorce dans les enquêtes quantitatives, les enquêtes de victimation, telles que l'enquête Cadre de vie et sécurité (CVS) (voir encadré), en dépit du progrès qu'elles représentent par rapport aux enquêtes précédentes, ne comportent pas d'interrogation sur les violences psychologiques. Lenquête Événements de la vie et santé (EVS) (voir encadré) inclut les violences psychologiques dans son champ d'analyse, en reprenant les formulations de l'enquête ENVEFF, mais l'imprécision des auteurs ne permet guère de cerner les violences psychologiques au sein du couple.

\section{Le viol conjugal}

Longtemps impensable, au nom du «devoir conjugal», le viol conjugal a été mis en lumière en 2000 (près d'une femme sur cent a déclaré en avoir été victime lors de l'enquête ENVEFF) et cette ampleur se retrouve en 2006 (enquête CVS). Dans la vie des couples contemporains, la sexualité semble tenir une place singulière dans le processus d'entente conjugale basé sur le respect de l'altérité, même si, en 2008, les décalages entre désirs masculin et féminin au sein des couples persistent (Bajos, Bozon,

3. Dans son pamphlet antiféministe Fausse route, 2003. 
2008). Lorsqu'il y a situation de violences conjugales, cette distance entre les motivations sexuelles des partenaires peut s'aggraver et transformer la négociation en sexualité imposée, par le chantage affectif, la menace, la force et la peur.

Le peu de reconnaissance concernant le viol conjugal a perduré jusqu'au vote de la loi de 2006 et le nombre annuel de viols commis par des conjoints ou concubins déclarés à la police est resté très faible: de 176 en 2000 , il est passé à 575 en 2006. Alors que l'ordre de grandeur du nombre de viols par des partenaires intimes estimé par les diverses enquêtes (voir encadré) atteint quelques dizaines de milliers ${ }^{4}$.

Si les dépôts de plainte demeurent rares, ces agressions sexuelles sont moins passées sous silence. L’enquête ENVEFF avait révélé l'ampleur du non-dit par les femmes sur les violences qu'elles subissaient (n'en ayant jamais parlé avant l'enquête), les femmes enquêtées plus récemment ont plus souvent parlé des violences subies antérieurement à leur interrogation; l'évolution la plus nette concerne les violences sexuelles commises par des proches. En 2006, au vu des données de l'enquête CVS (Tournyol du Clos, Le Jeannic, 2008) et de l'enquête Comportements sexistes et violences contre les filles (CSVF) (voir encadré), un tiers des victimes de violences sexuelles n'en avait pas parlé avant l'enquête, contre deux tiers en 2000 (ENVEFF). Toutefois, cette levée du silence porte davantage sur les violences subies dans l'enfance, la sexualité imposée par un partenaire demeurant un sujet relativement tabou.

\section{Les crimes de sang}

En France, jusqu'en 1975, en cas d'adultère, le meurtre commis par l'époux sur son épouse, ainsi que son complice, était excusable. Les circonstances aggravantes, en cas de coups et blessures, n'ont été introduites que dans les réformes les plus récentes du droit pénal.

La majorité des crimes de sang sont perpétrés par des proches. Les meurtres de femmes, essentiellement des meurtres conjugaux, sont encore masqués sous l'appellation romanesque de «crime passionnel», plus propre à émouvoir des jurys d'assises.

À l'été 2003, le meurtre de l'actrice Marie Trintignant par son compagnon Bertrand Cantat, chanteur du groupe Noir Désir, fait l'objet d'une très forte médiatisation liée à la notoriété des protagonistes. L'analyse de cette médiatisation montre les affrontements de l'opinion publique: antérieurement, seul le crime dit "passionnel» aurait été mis en avant; au contraire, une approche plus sociologique émerge: le couple Trintignant-Cantat est pris comme emblématique de la violence conjugale qui peut conduire jusqu'au meurtre (Houel et al., Sobota, 2008).

Les politiques, par le plan de lutte contre les violences - conçu avant le drame -, avaient déjà décidé de radicaliser les campagnes de sensibilisation. Afin qu'elles aient plus d'impact, la mort est mise en avant dans les spots et les slogans «Violences conjugales, parlons-en avant qu'il ne soit trop tard.». La simultanéité des faits donne un élan particulier à la prise en compte des violences conjugales, mais elle a aussi pour effet de transformer leur perception en masquant les violences au quotidien qui touchent le plus grand nombre (Jaspard, 2005).

À l'automne 2004, le débat public sur les violences conjugales se focalise de nouveau sur la dénonciation du silence face aux meurtres de femmes. Nombre d'articles de presse relèvent l'indifférence des pouvoirs publics face à ces crimes de sang et dénoncent l'absence de chiffres fiables sur le sujet. En effet, les statistiques officielles produites par les services de police ne sont pas sexuées. On peut penser que cette campagne des médias a contribué à la mise en œuvre de l'Étude nationale des décès au sein du couple (voir encadré). En 2006, on a enregistré 168 décès consécutifs aux violences d'un conjoint, dont 139 femmes et 29 hommes. Ces meurtres sont le fait d'époux, de concubins ou $\mathrm{d}^{\prime}$ «ex», mais n'incluent pas les autres intimes. Contrairement aux études canadiennes très détaillées quant au lien entre victime et auteur, les données françaises disponibles ne permettent pas ce niveau d'analyse. L'information

4. La définition juridique du viol conjugal est plus restreinte que celle de partenaire intime qui n'implique pas la résidence commune. 
a été diffusée en ces termes: «une femme meurt tous les trois jours sous les coups de son conjoint», tant par les acteurs politiques que par les différents supports médiatiques.

Les violences physiques: point de mire des violences conjugales

Les crimes conjugaux (viols et meurtres) sont aujourd'hui beaucoup plus mis en avant par les médias que les agressions les plus courantes, agressions qui constituent l'essentiel des situations de violences conjugales - violences verbales, menaces, dénigrements, contrôles et autres atteintes psychologiques. Les agressions physiques étant mieux répertoriées par les statistiques de la police et de la justice, l'appréhension des violences conjugales risque d'être à nouveau restreinte aux brutalités physiques.
De 2002 à 2006, le nombre de délits de violences conjugales (coups et blessures volontaires) enregistrés par les services de police est passé de 30661 en 2002 à 40507 en 2006 (Bonvoisin, 2006; Bauer, 2007). Ces statistiques montrent que les délits commis entre conjoints ont tendance à faire davantage l'objet de déclarations aux services de police et de gendarmerie. Cependant, elles ne peuvent pas rendre compte de l'évolution du phénomène, puisque les enquêtes ont montré l'ampleur du silence pesant sur ces violences, notamment des plus intimes.

Depuis 2007, grâce à l'enquête de victimation CVS, on dispose de statistiques annuelles sur les faits de violences physiques et sexuelles dans le cadre du couple. Globalement, on retrouve les mêmes tendances que dans l'enquête ENVEFF relativement

\section{LES ENQUÊTES DE VICTIMATION}

\section{- Enquête nationale sur les violences envers les femmes en France (ENVEFF)}

Cette enquête, coordonnée par I'Institut de démographie de I'Université Paris I (IDUP), a été réalisée en 2000 auprès d'un échantillon de 6970 femmes âgées de 20 à 59 ans par une équipe de recherche pluridisciplinaire. Les violences conjugales, incluant les violences verbales, psychologiques, physiques et sexuelles, subies pendant les 12 derniers mois sont cernées dans un module spécifique. Un questionnement sur les disputes est posé en début d'entretien. La notion de conjugal correspond à "partenaire intime" avec ou sans cohabitation.

\section{- Enquête Événements de la vie et santé (EVS)}

La Direction de la recherche, des études, de l'évaluation et des statistiques (DREES - ministère de la Santé et des Solidarités) et l'Institut national de la statistique et des études économiques (INSEE) ont réalisé cette enquête en 2005-2006 auprès d'un échantillon de 10000 hommes et femmes âgés de 18 à 75 ans. Les violences verbales, psychologiques physiques et sexuelles au cours des 24 derniers mois y sont appréhendées globalement et permettent de repérer les violences perpétrées par des proches, sans distinction du conjoint. Le même questionnement sur les disputes au sein du couple que dans l'enquête ENVEFF est posé (Cavalin, 2007).

\section{- Enquête Cadre de vie et sécurité (CVS)}

Cette enquête a été réalisée en 2007 (répétée en 2008) auprès d'un échantillon de 11250 hommes et femmes, âgés de 18 à 60 ans (pour les atteintes aux personnes), par l'Observatoire national de la délinquance et I'Institut national de la statistique et des études économiques (INSEE). Les violences physiques et sexuelles perpétrées par un conjoint au cours des 24 derniers mois y sont appréhendées dans un module auto-administré (audio CAPI) (Tournyol du Clos, Le Jeannic, 2008).

\section{- Enquête Comportements sexistes et violences contre les filles (CSVF)}

En 2006, l'Observatoire départemental des violences envers les femmes du Conseil général de SeineSaint-Denis et I'Institut de démographie de I'Université Paris I (IDUP) ont réalisé cette enquête auprès d'un échantillon de 1600 jeunes filles, âgées de 18 à 21 ans et habitant en Seine-Saint-Denis. Sur le modèle de l'enquête ENVEFF, un module cerne les violences perpétrées par un partenaire intime, ici un "petit ami » (Jaspard, 2007).

Les statistiques sur la mortalité liée aux violences conjugales s'appuient sur les données relevées par les services de police et de gendarmerie. Ainsi, I'École nationale de la statistique et de l'administration économique (ENSAE) et le ministère de I'Intérieur et de l'Aménagement du territoire ont réalisé, à la demande du ministère de la Parité, des "Recensements nationaux des morts violentes au sein du couple en 2003 et 2004 ». Une étude statistique conduite par la Délégation aux victimes du ministère de I'Intérieur en 2006 complète ces données. Cette recension statistique n'étant pas pérennisée, on peut relever l'absence de données fiables sur ce phénomène, les autres sources ne présentant pas le niveau de détail nécessaire à cette mesure. 
aux violences physiques et sexuelles. En l'absence d'étude quantitative plus ciblée sur la vie conjugale, ces enquêtes de victimation sont la seule source d'information. De fait, la non prise en compte des violences psychologiques dans ces enquêtes tend à privilégier une perception des violences conjugales en termes de «femmes battues». Les analyses de l'enquête ENVEFF ont mis en cause cette notion réductrice au profit de celle, plus adaptée, de «femmes en situation de violences conjugales» (Jaspard et al., 2003).

\section{Conclusion}

Les violences envers les femmes sont maintenant également définies comme forme de discriminations et comme obstacle à la pleine égalité de droits entre hommes et femmes, plus largement une atteinte à la citoyenneté des femmes. Mais l'autoritarisme des hommes et le maintien de leur position de pouvoir se manifestent toujours, notamment en matière de violences conjugales. L’enquête ENVEFF a permis nombre d'avancées, en réveillant l'intérêt des pouvoirs publics pour les phénomènes ainsi mis en évidence et en contribuant à ouvrir les perspectives des enquêtes de victimation. Celles-ci, désormais, traitent aussi des violences physiques et sexuelles dans le cadre du ménage, particulièrement entre conjoints, en s'efforçant d'adopter des protocoles de collecte nouveaux et adaptés. Malgré tout, certains écueils persistent, notamment le risque de réduire les violences conjugales aux seules violences physiques, d'amalgamer des violences conjugales et intrafamiliales, de confondre violence et conflit. Il est plus que jamais indispensable d'approfondir la réflexion scientifique et politique sur la pertinence et l'impact de la quantification sexuée des violences privées, en veillant à la précision de l'analyse sur ces sujets et en comparant avec prudence les résultats existants.

\section{Bibliographie}

Badinter E. (2003). Fausse route, Paris, Odile Jacob, 224.

Bajos N., Bozon M. (dir.) (2008). Enquête sur la sexualité en France: pratiques, genre et santé, Paris, La Découverte, 612.

Bauer A. (dir.) (2007). La criminalité en France, Rapport de l'Observatoire national de la délinquance 2007, Paris, CNRS Éditions, 573.

Bonvoisin V. (2006). Éléments de mesure des violences entre conjoints, in Institut national des hautes études de sécurité - Observatoire national de la délinquance, Rapport annuel 2006, Saint-Denis-La-Plaine, INHES-OND, 407-433.

Cavalin C., (2007). Les violences subies par les personnes âgées de 18 à 75 ans. Premiers résultats de l'enquête Événements de vie et santé (1/2l), Études et Résultats, DREES, $\mathrm{n}^{0} 598$, septembre.

Chetcuti N., Jaspard M. (dir.) (2007). Violences envers les femmes. Trois pas en avant deux pas en arrière, Paris, L'Harmattan, 319.

Délégation aux victimes (2007). Étude nationale des décès au sein du couple-Année 2006, Paris, Ministère de l'Intérieur et de l'Aménagement du territoire, 13.

ENSAE - Junior Études, Ministère de l'Intérieur et de l'Aménagement du territoire (2005). Recensement national de morts violentes survenues au sein du couple en 2003 et 2004, Paris, ENSAE, 40.

Fougeyrollas-Schwebel D., Jaspard M. (2003). Compter les violences envers les femmes. Contexte institutionnel et théorique de l'enquête ENVEFF, Cahiers du Genre, no 35, 35-58.

Houel A., Mercader P., Sobota H. (2008). Psychosociologie du crime passionnel, Paris, Presses universitaires de France, 256.

Jaspard M. (2007). Premiers résultats de l'enquête CSVF: des chiffres inquiétants, in Connaître et prévenir les comportements sexistes et violents, Observatoire départemental des violences envers les femmes du Conseil général de la Seine-Saint-Denis, Bobigny, 18-28. 
Jaspard M., (2005). Les violences contre les femmes, Paris, La Découverte, 122.

Jaspard M., Brown E., Condon S. (2003). Les violences envers les femmes en France. Une enquête nationale, Paris, La Documentation française, 374.

Tournyol du Clos L., Le Jeannic T. (2008). Les violences faites aux femmes, INSEE Première, $\mathrm{n}^{\mathrm{o}} 1180$, février. 